Artículos

\title{
Relación entre engagement y calidad de vida en el trabajo en funcionarios de establecimientos educacionales chilenos*
}

\author{
Relationship Between Engagement and Quality of Work Life in Chilean Educational Establishment Officials \\ Relação entre engagement e qualidade de vida no trabalho em funcionários de estabelecimentos de ensino chilenos
}

Carlos Javier Ossa Cornejo ${ }^{\text {a }}$

Universidad del Bio-Bio, Chile

cossa@ubiobio.cl

ORCID: https://orcid.org/0000-0002-2716-2558

DOI: https://doi.org/10.11144/Javeriana.cao34.recvtf

Andrés Eduardo Jiménez Figueroa

Universidad de Talca, Chile

ORCID: https://orcid.org/0000-0001-8324-0250

Recibido: 16 Marzo 2021

Aceptado: 19 Octubre 2021

Publicado: 30 Diciembre 2021

\section{Resumen:}

Este estudio analiza la relación entre engagement y calidad de vida en funcionarios de establecimientos educativos chilenos de las regiones de Maule y Nuble. Se aplicaron dos instrumentos: una adaptación del Cuestionario de calidad de vida laboral y el Utrecht Work Engagement Scale (UWES 17) que mide el nivel de engagement de la persona con su actividad laboral a un total de 241 funcionarios de ocho organizaciones escolares. Para la aplicación de los cuestionarios se realizó una versión online debido a la situación de pandemia por Covid-19. Los resultados muestran una tendencia a valorar positivamente los indicadores de calidad de vida en el trabajo y engagement, con alta correlación entre las dos variables. Se concluye que la relación entre estas variables es importante lo que permitiría promover estrategias de desarrollo organizacional en este tipo de instituciones, con miras a formular políticas de gestión de personas con un enfoque centrado en el equilibrio entre las necesidades personales y las organizacionales.

Códigos JEL: D21, I31.

Palabras clave: Engagement, educación, gestión, calidad, organizaciones.

\section{Abstract:}

The present study analyses the relationship between engagement and quality of life in educational establishment officials in the regions of Maule and Nuble in Chile. The study used two instruments: an adaptation of the Quality of Work Life Questionnaire, and the Utrecht Work Engagement Scale (UWES 17), which measures people's levels of engagement with work activities. 241 Chilean officials from 8 educational establishments participated in the study. The questionnaires were completed virtually due to the COVID-19 pandemic. The results show a tendency to positively value the quality of work life and engagement indicators, as well as a high correlation between the two variables. In conclusion, the relationship between these variables is important for furthering organisational development within educational institutions. It could also lead to the development of human resources management policies focused on a balance between personal and organisational needs.

JEL Codes: D21, I31.

Keywords: Engagement, education, management, quality, organizations.

\section{Resumo:}

Este estudo analisa a relação entre engagement e qualidade de vida em funcionários de estabelecimentos de ensino chilenos nas regiões de Maule e Ñuble. Dois instrumentos foram aplicados: uma adaptação do Questionário de Qualidade de Vida no Trabalho e a Utrecht Work Engagert4545ment Scale (UWES 17) que mede o nível de engagement da pessoa com sua atividade de trabalho para um total de 241 funcionários de oito organizações escolares. Para a aplicação dos questionários, foi feita uma versão online devido à situação pandêmica do Covid-19. Os resultados mostram uma tendência a valorizar positivamente os indicadores de qualidade de vida no trabalho e engagement, com alta correlação entre as duas variáveis. Conclui-se que a relação entre essas variáveis é importante, o que permitiria promover estratégias de desenvolvimento organizacional neste tipo de instituições, com vistas a formular políticas de gestão de pessoas com uma abordagem focada no equilíbrio entre as necessidades pessoais e organizacionais. Códigos JEL: D21, I31.

Palavras-chave: Engagement, educação, gestão, qualidade, organizações.

Notas de autor

a Autor de correspondencia. Correo electrónico: cossa@ubiobio.cl 


\section{Introducción}

Recientemente, factores como la calidad de vida, el bienestar personal y la salud mental han estado presentes tanto en el discurso social como en los medios de noticias. Si bien las mediciones de calidad de vida y salud en la población general muestran un panorama alentador, con disminución de indicadores de hábitos alimenticios negativos, de riesgo sexual, de tabaquismo y alcoholismo, al relacionar la calidad de vida y su impacto en la salud, con el trabajo en educación, se encuentran pocos estudios en el ámbito educativo. Los indicadores de la carga laboral y el agotamiento experimentado por parte de docentes presentan niveles muy altos, sumando a ello escasos espacios de autocuidado, así como de apoyo de la gestión directiva (Miño, 2016; Jomuad, Antiquina, Cericos et al., 2021), lo cual repercutiría negativamente en el desarrollo profesional y personal de los docentes.

La tarea del educador y las condiciones laborales que se presentan en gran parte de los establecimientos educativos chilenos se pueden considerar factores generadores de estrés negativo, insatisfacción laboral, patologías de salud física y mental, que afectan el desempeño laboral de los docentes y pueden representar largas licencias médicas y hasta la deserción del campo laboral (González, 2020, Alonso, 2014; Erazo, 2015; Miño, 2016).

Por lo anterior es relevante contar con un espacio laboral que otorgue apoyos, orientaciones y condiciones de cuidado para sus funcionarios, de manera que se promuevan contextos que optimicen el desarrollo personal y profesional, así como la motivación laboral (Heckmann, Sandoval \& Arroyo-Jofré, 2021). Estos elementos del contexto organizacional pueden generar bienestar o malestar en las personas y, por lo tanto, incidir en su desempeño e implicación con el trabajo (Hipólito, Masson, Monteiro et al., 2017).

La calidad de vida laboral en instituciones educativas es un tema poco investigado en Chile, aun cuando en los últimos cinco años se han realizado estudios que buscan conocer las características de la calidad de vida en docentes de educación secundaria (Canales-Vergara, Valenzuela-Suazo \& Luengo-Machuca, 2018; Castilla-Gutiérrez, Colihuil-Catrileo, Bruneau-Chávez \& Lagos-Hernández, 2021; Miño 2016). Aunque teóricamente se ha planteado su relación con el engagement (Knight, Patterson \& Dawson, 2016; Salanova \& Llorens, 2016), no se encontraron investigaciones en el terreno empírico de la interrelación de estas variables, por lo que se desconoce cómo se pueden integrar en la gestión de las instituciones de educación secundaria.

Si bien hay estudios que han mostrado una relación positiva y significativa entre engagement y calidad de vida laboral a nivel latinoamericano, éstas se han llevado a cabo principalmente en organizaciones comerciales o de salud (Arenas \& Andrade, 2013; Pérez-Zapata, Peralta-Montecinos \& Fernández-Dávila, 2014; Ospina \& Meneghel, 2016; Redondo, 2015; Vesga, García, Forero et al., 2021), y escasamente en instituciones educativas, que han sido además, de educación superior (Rubio, 2018; Ruiz de Chávez, Pando, Aranda et al., 2014; Toscano-del Cairo, Vesga-Rodríguez \& Avendaño, 2020); dejando de lado lo que ocurre en instituciones de educación escolar.

Esto es relevante pues la gestión escolar es un área que se ha instalado de manera muy reciente en el ámbito educativo y, muchas veces, sin el éxito esperado (Bodero, 2014; García, Juárez \& Salgado, 2018) porque la dinámica entre necesidades organizacionales y educativas no logran tener la vinculación adecuada, necesitándose modelo que se apliquen en forma pertinente al campo educativo, y no solo transfiriendo desde la economía o la administración (Garbanzo, 2015).

Por tanto, este estudio busca analizar la relación entre el engagement y la dimensión calidad de vida del trabajo en una muestra de funcionarios de establecimientos educativos chilenos. Se formula la hipótesis de la existencia de una correlación estadísticamente significativa, positiva y de intensidad fuerte entre las dos variables. Asimismo, se busca plantear una discusión sobre la necesidad de fortalecer estrategias de gestión eficientes en el campo educativo para el desarrollo de instituciones saludables. 


\section{Calidad de vida y educación}

La calidad de vida es un tema relevante en la actualidad, situándose como uno de los factores centrales de desarrollo humano (Miño, 2016; Ossa et al., 2015); es un concepto multidimensional que contiene elementos de bienestar, políticas sociales, dimensiones materiales y no materiales, objetivas y subjetivas, individuales y colectivas (Silva, Picinin, Pilati et al., 2021). Las propuestas de aplicación del concepto calidad de vida en la educación han contribuido a que los centros escolares puedan conocer mejor su realidad, juntamente con aspirar a mejorarla.

En el primer Censo de calidad de vida docente (Eduglobal, 2012) se logró visibilizar el impacto negativo que tenían sobre la calidad de vida de los profesores las exigencias y el rol multidimensional de los docentes, a través de tareas como atención de apoderados y alumnos, labores administrativas, horas no lectivas, las cuales se suman a condiciones de trabajo que les impedían de disponer del tiempo necesario para descansar o realizar actividades extralaborales.

Estos antecedentes se complementan con un estudio que respalda la existencia de una fuerte relación entre las condiciones laborales, el bienestar y la salud de los docentes (Ribeiro, Dos Santos, Scorsolini-Comin, \& Dalri, 2020), lo que contribuye a poner en relieve la complejidad y multidimensionalidad del concepto calidad de vida laboral, pues esta se configura de una dimensión material (que puede estar asociada a las condiciones en las cuales se desempeña el trabajo) y una dimensión asociada al bienestar subjetivo, el cual está focalizando en aspectos afectivos-emocionales, vinculándose a estados de ánimo de las personas y a cómo y porqué experimentan su vida de forma positiva (Gutiérrez, Tomás \& Pastor, 2020; Miño, 2016).

Por otro lado, en la investigación efectuada por Canales-Vergara et al. (2018) en la ciudad de Concepción, Chile, se concluye que los profesores de liceos y colegios públicos están medianamente satisfechos con su calidad de vida en el trabajo, siendo la prexistencia de trastornos en salud mental la variable que presenta mayor asociación con una percepción más baja en su calidad de vida en el trabajo. Complementariamente, la Superintendencia de Seguridad Social de Chile (2017), específicamente en el área educativa, identifica que las/los profesionales docentes presentan problemáticas asociadas al área de salud física como enfermedades músculo esqueléticas y disfonía, y en el área de salud mental enfermedades como trastornos adaptativos.

Según Guerrero-Barona, Gómez, Moreno-Manso et al. (2018), las principales fuentes de estrés para los profesores corresponden a la indisciplina y la falta de respeto de por parte de alumnos, la falta de interés y escasa motivación de los estudiantes, junto a la falta de colaboración y apoyo por parte de las familias. Indican también el escaso reconocimiento de los padres por su labor docente, vinculando todos estos aspectos con los niveles de salud mental del profesorado, en que 36,3\% de los mismos presentan riesgo de salud mental o patología psiquiátrica y que el $63,7 \%$ presentan una buena salud mental.

Factores como la violencia en la escuela también han sido referido por Gómez, Padilla, Rodríguez et al. (2010), constatando que las agresiones, tanto físicas como verbales contra el profesor, las amenazas con armas, el tráfico y el consumo de drogas en la escuela, se encuentran asociados a trastornos mentales en los docentes.

Otras investigaciones como la llevada a cabo por Cuadra, Jorquera \& Pérez (2015) reportan información respecto a las teorías subjetivas sobre la salud laboral docente, las cuales se relacionan con los significados subjetivos que los profesores otorgan a la profesión, a la enseñanza y el aprendizaje de sus estudiantes, señalando que las características de su estructura y contenido se orientan más bien a una inhibición del autocuidado de su salud laboral. De este modo, plantean que para la promoción de la salud laboral se requiere de la consideración de diversos elementos, entre los cuales destacan la capacitación de los profesores en torno a su salud, la existencia de un clima social escolar nutritivo, la disminución de la sobrecarga de trabajo, junto a la presencia de una cultura organizacional en pro de la salud laboral y de políticas educativas que la consideren. Cabe señalar también que en la literatura revisada no se presentan reportes de investigación de la salud mental en asistentes de la educación, personal de apoyo profesional y técnico, importantísimo en las tareas escolares, desconociéndose los factores de riesgo para este grupo específico. 
En cuanto a los resultados obtenidos en la evaluación de dimensiones de ambiente físico -entorno y espacio físico del lugar de trabajo- así como de percepción de las prestaciones recibidas -cumplimiento de convenios laboral, promoción y formación- se observan en un nivel medio de satisfacción. Al respecto, investigaciones como la de Bedoya, Carrillo, Severiche et al. (2018) respaldan este resultado señalando que las mayores insatisfacciones de los docentes se relacionan con los espacios físicos y el salario.

La calidad de vida enfocada en el ambiente laboral ha sido considerada como la aplicación de las dimensiones de la calidad de vida en los entornos de trabajo, considerando el bienestar del empleado, las condiciones materiales de la organización, las características del puesto de trabajo, aspectos de riesgo nocivo, la estabilidad laboral, el salario, entre otros (García, González, Aldrete et al., 2014; Patlán, 2016).

La calidad de vida en el trabajo se conceptualiza como una filosofía de la organización que permite promover la eficiencia en el desempeño y la motivación del trabajador, a través de estrategias corporativas y de espacios de participación, promoviendo el bienestar personal, las condiciones de salud y motivación laboral (Hipólito et al., 2017). Sin embargo, se ha planteado también que es un concepto de difícil conceptualización debido a que, en muchas ocasiones, se le ha derivado al ámbito laboral a partir de las conceptualizaciones de los modelos de calidad de vida tradicionales, o desde aquellos que lo han relacionado con la salud, no considerando todas las dimensiones pertinentes del ámbito organizacional (Ribeiro et al., 2020). Por otra parte, quizás por la complejidad del ámbito organizacional y laboral en los últimos años se han desarrollado definiciones e instrumentos para medir calidad de vida en el trabajo con diversas características y factores (Patlán, 2016).

La implementación de programas de promoción de calidad de vida en las organizaciones debe verse como una estrategia de desarrollo más que como una política asistencial que solo busca compensaciones. Una mirada integral de la calidad de vida del trabajo lleva a fortalecer la experiencia laboral, permitiendo no solo aumentar la motivación y reducir el estrés de los trabajadores, sino además fortalecer aspectos de la cultura organizacional como la participación, la igualdad de género y la colaboración, e incluso mejorar el equilibrio entre vida laboral y familiar (Silva et al., 2021).

Horn \& Murillo (2016) exponen que las acciones desarrolladas por las figuras directivas -forma de liderazgo- en el contexto educativo influyen en el compromiso que presentan las/los docentes. De aquí se infiere que los directivos podrían favorecer el proceso de aprendizaje de los estudiantes interviniendo la variable compromiso laboral de las/los docentes.

Para Salanova, Martínez \& Llorens (2014) los recursos y las prácticas más importantes para el desarrollo de organizaciones efectivas son: (1) el desarrollo de habilidades, (2) salud psicosocial, (3) conciliación trabajo familia, (4) prevención del acoso laboral, (5) desarrollo de carrera, (6) equidad percibida, (7) comunicación e información organizacional, y (8) responsabilidad social. Los autores demuestran que estas prácticas se relacionan positivamente con trabajadores y equipos más eficientes, resilientes y comprometidos (engaged), que a su vez tienen buen desempeño, produce resultados organizacionales de mayor calidad y brindan mejores servicios.

Los factores psicosociales y de bienestar subjetivo son primordiales para motivar el compromiso con la institución y su desarrollo (Parada \& Pérez, 2014), planteándose el concepto de engagement para identificar las conductas de compromiso, tanto a nivel individual como a nivel organizacional, de las personas que trabajan en la organización (Fundación Chile, 2018). Los empleados que presentan un mayor nivel de este constructo disfrutan de su trabajo, experimentan una conexión con este, se sienten con la capacidad de enfrentar las demandas, impulsan a los otros y en general obtienen mejores resultados (Carmona, Schaufelli \& Salanova, 2019). De esta forma, como lo señala Marsollier (2021), el estudio del engagement es de vital importancia para que las organizaciones puedan adaptarse a las transformaciones y sobrevivir en el contexto de cambio laboral. 


\section{Engagement y calidad de vida}

La salud ocupacional, tratando de comprender la relación entre los sujetos y su trabajo, utilizaba el término adicto al trabajo para denominar a aquellos trabajadores que invertían gran cantidad de tiempo en las tareas laborales. A estos trabajadores se les veía desde una connotación negativa, debido a que solo se enfocaban en las consecuencias dañinas para la salud, donde posteriormente se dio paso al estudio del síndrome de burnout (Contreras, 2015). Sin embargo, desde un cambio de paradigma de la psicología, dirigida hacia una aproximación positiva, se comienza a observar que algunos trabajadores se enfocaban en su trabajo sin presentar consecuencias negativas, de este modo, el concepto de engagement comienza a jugar un rol crucial para el desarrollo laboral de las personas (Salanova, Llorens, Cifre et al., 2012; Salanova et al., 2014).

El constructo engagement surge en la década de 1990 con el movimiento de la psicología positiva como elemento que busca considerar el aspecto y la potencialidad humana (Salanova et al., 2012; Cánovas \& Chiclana, 2018). Con el surgimiento de este constructo hubo un cambio de paradigma generalizado, donde las investigaciones comienzan a orientarse a la promoción de la salud, ya que esto no solo traería beneficios para la persona, sino que la organización, podría verse beneficiada por la mayor calidad laboral de aquellos que experimentarán el trabajo como un lugar que se preocupa por ellos (Bakker \& Rodri\#guez-Mun\#oz, 2012; Marsollier, 2021).

De este modo, el engagement es uno de los elementos que más puede beneficiar a la organización, pues permitiría contar con empleados altamente implicados en el trabajo realizado. Los sujetos que experimentan este estado presentan un sentimiento de vigorosidad hacia el trabajo (energía, resiliencia y el deseo por dar lo mejor de sí en el trabajo), dedicación (verse envuelto en lo que se realiza, entusiasmo, orgullo y retarse en el trabajo) y absorción (concentración y bienestar en el trabajo) trayendo beneficios tanto en sus trabajadores como en las organizaciones (Ventura, Salanova \& Llorens, 2015, Bakker, Albrecht, \& Leiter, 2011). Esta variable ha sido estudiada ampliamente en el contexto organizacional y se ha asociado a otras variables, siendo el enriquecimiento trabajo-familia uno de los predictores por excelencia (Ling, Fang, Brought et al., 2010).

En este sentido, la primera descripción aportada de engagement se enmarca en la comprensión de aprovechamiento físico, cognitivo y emocional de los roles que desempeñan los sujetos en su trabajo (Schaufeli \& De Witte, 2017). Ha sido descrito también como un estado motivacional positivo, caracterizado por tres variables fundamentales del trabajador: vigor, dedicación y absorción (Carmona et al., 2019). El vigor se refiere principalmente al esfuerzo realizado en las labores y la persistencia ante las dificultades que estas puedan presentar; la dedicación se caracteriza por lo involucrado que pueda estar un sujeto en su trabajo, lo que se manifiesta en características como entusiasmo y aspiración; finalmente, la absorción se refiere a la concentración como estado positivo en las tareas organizacionales, así, el trabajador tiene la sensación de que el tiempo invertido en las labores pasa rápidamente.

Los trabajadores que tienen estas características, o más bien entendidos como trabajadores engaged, tienen una serie de características positivas, tales como sentido de pertenencia hacia la organización, son fieles a la misma, rinden más allá de lo que se les exige, tienen un fuerte grado de conexión energética y afectiva con su trabajo, son proactivos, buscan y aceptan responsabilidades, así como retroalimentación referente a estas, plantean alternativas de mejora, y tienen valores que coinciden con los de la organización (Lorente \& Vera, 2010).

Uno de los estados de los cuales más se beneficia la organización es el contar con empleados altamente envueltos en el trabajo realizado, lo que se conoce como el estado de engagement.

La Psicología Organizacional Positiva (POP) ha intentado dar cabida y respuesta a estos temas mediante la introducción y estudio de diferentes variables, todo esto con el fin de generar un equilibrio y un óptimo desarrollo en las empresas, de manera que los empleados puedan conseguir su propio bienestar social y emocional, para así desempeñarse de manera productiva y comprometida con las metas de la organización (Forbes, 2013). Es importante considerar la relación que se establece entre esta variable y otros factores que 
también ejercen influencia en los empleados; en este punto se encuentra el estudio de los factores psicosociales, los cuales comprenden el estado del sujeto y el entorno de trabajo que pueden causar efectos positivos o perjudiciales tanto en el personal como en la organización (Salanova \& Llorens, 2016).

Tomados en conjunto, algunos de los factores psicosociales se relacionan positivamente con el engagement y actúan como predictores de este, por ejemplo, ambas variables se asocian al apoyo social y a los recursos que permiten el crecimiento personal, la reducción de las demandas y el cumplimiento de los objetivos (Knight, Patterson \& Dawson, 2016; Arenas \& Andrade, 2013). Así, la salud de los/as trabajadores/as en términos de bienestar se explica según Acosta, Cruz, Salanova et al. (2015) como el cuerpo de una organización, es decir, su estructura, las creencias de los procesos de comunicación y la distribución del trabajo y por último los objetivos y metas de la organización. Por el contrario, existe una organización tóxica, entendida como aquella donde la interacción de las personas con su trabajo y con los demás, tiene efectos psicosociales nocivos y perjudiciales, tanto para la organización como para los/as trabajadores/as que la integran, afectando de tal manera el bienestar psicosocial y la productividad de la organización (Salanova et al., 2012; Salanova \& Llorens, 2016).

De esta forma, la influencia que tienen tanto el engagement como los factores psicosociales para potenciar la calidad de vida de los integrantes de la organización deriva en la necesidad por parte de aquellas de invertir recursos y esfuerzos para tener a sus integrantes sanos física y mentalmente, además de mantenerlos motivados para afrontar los cambios y de este modo prosperar (Martínez, Youssef-Morga, Chambel et al., 2019; Cánovas \& Chiclana, 2018).

\section{Método}

El presente estudio tiene una finalidad descriptiva para establecer, desde un marco empírico, la relación entre las variables estudiadas. Con este propósito se desarrolló un diseño descriptivo correlacional, no experimental y transeccional.

\section{Participantes}

Los participantes fueron 241 funcionarios de establecimientos educativos de las regiones de Maule y Nuble, el $83 \%$ son mujeres ( 241 personas) mientras que el $17 \%$ son hombres ( 40 personas). La edad de los participantes varía entre los 22 y los 78 años ( $\mathrm{M}=38$, D.E. 10,33); el 57\% (138 personas) son de establecimientos municipales, y el 43\% (103 personas) son de establecimientos particular subvencionados.

\section{Instrumentos}

Se utilizaron dos instrumentos, en primer lugar, una versión adaptada del Cuestionario de Calidad de Vida en el trabajo (Patlán, 2016), el que se encuentra orientado a evaluar dimensiones de calidad de vida existentes en las organizaciones. El instrumento original presenta 117 ítems y 17 factores con una confiabilidad adecuada (alfa de Cronbach de 0,750).

Para este estudio se seleccionaron siete factores y 49 ítems relacionados con aspectos individuales, de la organización, del ambiente de trabajo y del entorno sociolaboral. Los factores específicos son desarrollo laboral y profesional, bienestar en el trabajo, condiciones y medio ambiente de trabajo, seguridad y salud en el trabajo, autonomía y control en el trabajo, relaciones interpersonales, y por último, apoyo organizacional. Las respuestas se organizan en una escala Likert de cinco alternativas que van desde nada de acuerdo hasta muy de acuerdo, con una alternativa neutra (ni acuerdo ni desacuerdo). 
El segundo instrumento es la Utrecht Work Engagement Scale (UWES 17) de Schaufeli et al. (2002) que mide el nivel de engagement de la persona con su actividad laboral, compuesto por 17 ítems organizados en tres dimensiones: vigor, absorción y dedicación, con niveles de confiabilidad sobre 0,70 , lo que se considera adecuado (Ávila, Portalanza y Duque, 2017). Las respuestas se organizan, igualmente, en una escala Likert de cinco alternativas que van desde nada de acuerdo hasta muy de acuerdo, con una alternativa neutra (ni acuerdo ni desacuerdo).

\section{Procedimientos}

En primer lugar se realizó un contacto con los directores de los establecimientos para motivarlos en su participación, luego se realizó un documento informativo de los objetivos del estudio para que se compartiera en el establecimiento y se lograra participación de los funcionarios. Para la aplicación de los cuestionarios se realizó una versión online debido a la situación de pandemia por Covid-9, que los participantes respondían desde sus equipos computacionales o smartphone. El cuestionario incluía un consentimiento informado de participación en la parte inicial del instrumento. Los resultados se ordenaron en una base de datos y fueron analizados mediante el software estadístico SPSS v. 21, y se aplicaron estadígrafos descriptivos (tendencia central, dispersión y distribución), de distribución de frecuencia, y de correlación ( $\mathrm{r}$ de Pearson).

\section{Resultados}

Los valores de confiabilidad de los instrumentos reportados por los participantes del estudio es buena, como se observa en la tabla 1. Destacan los valores de la escala de calidad de vida del trabajo y sus dimensiones, que se encuentran todas con un nivel de confiabilidad bueno y excelente.

TABLA 1

Valores de confiabilidad de los instrumentos

\begin{tabular}{lcc}
\hline \multicolumn{1}{c}{ Variables y dimensiones } & Ítems & Alfa \\
\hline Engagement total & 17 & 0,916 \\
Vigor & 6 & 0,846 \\
Absorción & 6 & 0,754 \\
Dedicación & 5 & 0,814 \\
CVT total & 49 & 0,962 \\
Desarrollo profesional & 8 & 0,908 \\
Bienestar & 7 & 0,900 \\
Condiciones laborales & 6 & 0,871 \\
Seguridad trabajo & 7 & 0,874 \\
Estabilidad & 5 & 0,912 \\
Relaciones interpersonales & 7 & 0,896 \\
Apoyo organización & 8 & 0,925 \\
\hline
\end{tabular}

Fuente: elaboración propia.

El análisis descriptivo señala que las variables principales de engagement y calidad de vida en el trabajo se encuentran con un promedio que tiende a valoraciones positivas, ya que la escala va de 1 que corresponde a muy en desacuerdo, hasta 5 que corresponde a muy de acuerdo, observándose baja dispersión en ambas. Dicha tendencia a la alta valoración de las variables podría estar influida por la deseabilidad social. Este aspecto es relevante pues gran parte de las escalas utilizadas para medir estos procesos psicosociales se estructuran con base en autorreportes y son sensibles a la deseabilidad. Ver tabla 2. 
TABLA 2

Valores del análisis descriptivo de las variables

\begin{tabular}{|c|c|c|c|c|c|c|}
\hline $\begin{array}{l}\text { Variables y } \\
\text { dimensiones }\end{array}$ & $\begin{array}{c}\text { Míni- } \\
\text { mo }\end{array}$ & $\begin{array}{l}\text { Máxi- } \\
\text { mo }\end{array}$ & Media & $\begin{array}{l}\text { Desviación } \\
\text { estándar }\end{array}$ & $\begin{array}{c}\text { Asime- } \\
\text { tría }\end{array}$ & $\begin{array}{l}\text { Curto- } \\
\text { sis }\end{array}$ \\
\hline Engagement total & 1,24 & 5,00 & 4,12 & 0,596 & $-1,221$ & 1,404 \\
\hline Vigor & 1,00 & 5,00 & 4,06 & 0,697 & $-1,043$ & 1,601 \\
\hline Absorción & 1,50 & 5,00 & 3,96 & 0,650 & $-0,737$ & 0,633 \\
\hline Dedicación & 1,00 & 5,00 & 4,33 & 0,647 & $-1,349$ & 1,735 \\
\hline CVT total & 2,05 & 4,85 & 3,98 & 0,555 & $-0,761$ & 0,030 \\
\hline $\begin{array}{l}\text { Desarrollo } \\
\text { profesional }\end{array}$ & 1,38 & 5,00 & 3,94 & 0,860 & $-0,794$ & $-0,077$ \\
\hline Bienestar & 1,57 & 5,00 & 4,29 & 0,677 & $-1,186$ & 1,357 \\
\hline $\begin{array}{l}\text { Condiciones } \\
\text { laborales }\end{array}$ & 1,00 & 5,00 & 4,12 & 0,739 & $-0,862$ & 0,781 \\
\hline Seguridad trabajo & 1,43 & 5,00 & 3,94 & 0,929 & $-0,521$ & $-0,800$ \\
\hline Estabilidad & 1,00 & 5,00 & 3,89 & 0,976 & $-0,969$ & 0,490 \\
\hline $\begin{array}{l}\text { Relaciones } \\
\text { interpersonales }\end{array}$ & 1,33 & 5,00 & 4,24 & 0,796 & $-1,405$ & 1,940 \\
\hline $\begin{array}{l}\text { Apoyo } \\
\text { organización }\end{array}$ & 1,25 & 5,00 & 4,10 & 0,777 & $-1,007$ & 0,847 \\
\hline
\end{tabular}

Fuente: elaboración propia.

Respecto al engagement, si bien la distribución es no homogénea con valores medianamente altos de asimetría y curtosis con valores hasta $\pm 1,5$, los resultados permiten suponer una distribución normalizada (Tabachnik \& Fidell, 2001). Las dimensiones que componen esta variable presentan valores promedios que tienden hacia la valoración positiva, destacando la de dedicación, que muestra el nivel de interés y entusiasmo en las tareas. Cabe señalar que las dimensiones de dedicación y de absorción, referidas al involucramiento y concentración en el trabajo (Pujol-Cols \& Arraigada, 2019), presentan una distribución leptocúrtica, con valores de curtosis mayores a $\pm 1,5$.

La valoración positiva del engagement en funcionarios escolares no es algo sorprendente, muchos de ellos se encuentran atraídos por el trabajo docente, evidenciando la llamada vocación docente (Larrosa, 2010). Sin embargo, en muchas ocasiones esta vocación e interés por las tareas docentes se ven influidas negativamente por situaciones organizacionales y fallas en la gestión escolar.

La variable calidad de vida en el trabajo total se observa con un promedio que tiende a la valoración medianamente positiva, con baja dispersión y una distribución adecuada de asimetría y curtosis $( \pm 1)$, lo que permite suponer una distribución normalizada de los datos. De las dimensiones que componen esta variable se observa que bienestar es la que presenta mayor valoración, lo que daría cuenta de una sensación de agrado y satisfacción con el lugar de trabajo, mientras que la menos valorada es estabilidad, relacionada con la seguridad de permanencia en el puesto de trabajo (Patlán, 2016). Asimismo se observa que la mayoría de las dimensiones exhiben una distribución dentro de los parámetros esperados $( \pm 1,5)$ para una distribución normalizada; la excepción es la dimensión de relaciones interpersonales, que presenta una distribución leptocúrtica, con alto valor de curtosis.

En cuanto a la relación entre las variables, en la tabla 3 se observa que existe una correlación significativa, positiva y medianamente fuerte entre el engagement y la calidad de vida laboral $(r=0,672 p<0,01)$, lo que respalda la vinculación entre ambas variables para potenciar el desarrollo positivo de funcionarios de establecimientos educacionales. Este resultado coincide con los de estudios realizados en otras áreas (Rubio, 2018; Ruiz de Chávez et al., 2014; Toscano-del Cairo et al., 2020), pero con indicadores de mayor fortaleza en la relación entre ambas variables $(0,62)$ en comparación con las correlaciones de estudios anteriores (entre $0,38$ y 0,55$)$. 
TABLA 3

Correlaciones entre las variables

\begin{tabular}{lcccc}
\hline \multicolumn{1}{c}{$\begin{array}{c}\text { Variable } / \\
\text { dimensiones }\end{array}$} & $\begin{array}{c}\text { Engagement } \\
\text { total }\end{array}$ & Vigor & Absorción & Dedicación \\
\hline CVT total & 0,672 & 0,650 & 0,512 & 0,675 \\
Desarrollo profesional & 0,611 & 0,600 & 0,458 & 0,623 \\
Bienestar & 0,745 & 0,706 & 0,541 & 0,774 \\
Condiciones laborales & 0,501 & 0,467 & 0,381 & 0,513 \\
Seguridad trabajo & 0,235 & 0,247 & 0,187 & 0,225 \\
Estabilidad & 0,406 & 0,428 & 0,321 & 0,356 \\
Relaciones & 0,574 & 0,574 & 0,406 & 0,589 \\
interpersonales & 0,602 & 0,596 & 0,441 & 0,610 \\
Apoyo organización & Fuente: elaboración propia. & \\
\hline Nota: todos los valores son significativos al nivel del p-value <0,01. &
\end{tabular}

Se puede observar, además, en la vinculación entre las variables y sus dimensiones, que todas presentan correlaciones positivas y estadísticamente significativas $(\mathrm{p}<0,01)$, destacando la dimensión de bienestar de la escala calidad de vida en el trabajo, que presenta las correlaciones más altas, con la escala de engagement y sus dimensiones, solo con excepción de la dimensión de absorción. Por otro lado, la dimensión que presenta menor relación es la de seguridad en el trabajo, que muestra correlaciones débiles con la escala de engagement y sus dimensiones. Asimismo, la dimensión de estabilidad es la segunda dimensión con menores relaciones con engagement.

La mayoría de las dimensiones de la variable calidad de vida en el trabajo presenta relaciones significativas con engagement, presentando correlaciones sobre 0,5 , excepto con seguridad en el trabajo y estabilidad. Las dimensiones de vigor y dedicación son las que presentan mayores niveles de correlación con las dimensiones de calidad de vida en el trabajo.

Estas correlaciones permiten reforzar los hallazgos reportados en otros estudios (Acosta et al., 2015; Salanova \& Schaufeli, 2009; Salanova et al., 2014), y dan cuenta de la importancia de trabajar en forma integral y sistémica los procesos organizacionales y de gestión para fomentar la satisfacción y la motivación hacia el desempeño laboral y la implicación al trabajo.

Las relaciones positivas y moderadamente fuertes entre engagement y calidad de vida laboral darían cuenta de un componente emotivo y de satisfacción de necesidades que se debe considerar en los planes de mejoramiento de la calidad de vida laboral, reforzando la idea de que se las instituciones se deben a las personas que las constituyen.

\section{Conclusiones}

Las organizaciones no se logran comprender bajo la mirada simplista de una suma de partes que trabajan en conjunto; por el contrario, son más bien sistemas vivos y dinámicos, que experimentan transformaciones y apuntan sus objetivos hacia el logro de resultados (García et al., 2018).

Se han realizado estudios sobre engagement tanto para el desarrollo de equipos de trabajo efectivos como para analizar su relación con el burnout, los climas laborales y la calidad de vida laboral, sin embargo, dichos estudios están orientados hacia el sector de salud, empresarial o al de educación superior, más que hacia el sistema escolar (Fundación Chile, 2017; Parada \& Pérez, 2014). El desarrollo de los recursos humanos en las organizaciones escolares y la calidad de vida en el trabajo han recibido poca atención lo que no ha permitido promover estrategias efectivas de desarrollo organizacional en este tipo de instituciones, tanto en Latinoamérica como en Chile, existiendo escasas investigaciones sobre esta temática en la región.

Los datos recabados permiten reforzar la relación entre engagement y calidad de vida señalada en diversas investigaciones (Arenas \& Andrade, 2013; Pérez-Zapata et al., 2014; Ospina \& Meneghel, 2016; 
Redondo, 2015; Vesga et al., 2021), dando cuenta de que las condiciones organizacionales y personales en establecimientos educacionales pueden ser manejadas en forma integral, para alcanzar un contexto que brinde la motivación necesaria para que los funcionarios se desempeñen en forma efectiva y satisfactoria, dado que tradicionalmente la labor en establecimientos educativos se ha considerado una tarea estresante y alienante (Miño, 2016).

Respecto a los hallazgos de la investigación, cabe destacar que se logró establecer una vinculación entre los aspectos específicos de las variables engagement y calidad de vida, pues otros estudios han trabajado dicha relación de manera global (Ruiz de Chávez et al., 2014; Toscano-del Cairo et al., 2020), lo que permite generar una mirada más específica al fenómeno y establecer apoyos concretos. En función de ello, destaca el aspecto emocional que se puede detectar en esta vinculación, al observar que el bienestar del funcionario es la dimensión que presenta mayores correlaciones con el engagement. Esto es importante puesto que el aspecto emocional es un ámbito muy difuso en el campo educativo, siendo valorado como un elemento moral relevante para el aprendizaje, pero escasamente analizado y trabajado en los centros educativos (González, Aranda \& Berrocal, 2010).

Lo anterior se ve reforzado además por la fuerte relación encontrada entre la dimensión de dedicación (o compromiso) del engagement con la calidad de vida laboral, que también presenta una fuerte carga emotiva de la persona hacia su lugar de trabajo. El compromiso es uno de los elementos cruciales de las estrategias de fidelización de empleados (Cruz, 2019), por lo cual podrían incorporarse como estrategias para el fortalecimiento del recurso humano en la gestión escolar. Es muy importante cambiar el foco en la gestión escolar, habitualmente centrada en los resultados, hacia la valoración de los procesos humanos como recurso de mejoramiento y de promoción de calidad.

Por otra parte, el elemento de la calidad de vida en el trabajo menos vinculado con el engagement es el de seguridad en el trabajo, lo que podría explicarse posiblemente, debido a que los funcionarios de centros educativos relacionan su desempeño con elementos subjetivos y de interacción social, antes que con elementos físicos y de infraestructura (Van den Berg, Mastenbroek, Scheepers et al., 2017; Jiménez, Jara \& Miranda, 2012). Esto se puede sustentar además, en que seguridad en el trabajo es la segunda dimensión menos valorada de la calidad de vida en el trabajo, por parte de los participantes, después de estabilidad laboral.

Si bien, el engagement puede ser un factor protector ante la aparición del síndrome de burnout, las variables presentes entre los constructos no son opuestos absolutos, pudiendo coexistir entre sí dependiendo de elementos como la naturaleza de la tarea desarrollada o la significancia social que esta presenta. En este sentido, el engagement es un elemento protector, pero pueden darse situaciones en que el sujeto experimente ambos estados (Chávez, Moreno, Beltrán et al., 2014).

Dentro de las limitaciones del estudio se puede señalar la baja variabilidad de la muestra y su baja representatividad a nivel nacional, lo que restringe la generalización de los resultados. Se puede mencionar también que las escalas presentarían sensibilidad a la deseabilidad social, dado que dos de las tres dimensiones de engagement, y dos de las siete dimensiones de calidad de vida en el trabajo muestran una distribución que no sería normalizada a juzgar por los valores de la curtosis. Esto estaría señalando una tendencia a responder en forma positiva en dichas dimensiones, debido a que sería lo políticamente correcto.

Finalmente se espera que este estudio aporte al efecto que tienen las prácticas organizacionales positivas en los/as trabajadores/as de establecimientos educativos, y cómo estas prácticas se configuran equilibrando aspectos personales y organizacionales, para que las organizaciones educativas consideren estos elementos como desafíos futuros y busquen de manera activa la realización de cambios pertinentes desde la gestión escolar, que permitirían maximizar tanto los objetivos organizacionales como los de sus trabajadores.

Se busca aportar con nuevos conocimientos teóricos sobre el efecto que tienen las prácticas organizacionales positivas en la promoción de estados positivos en el empleo y la vida del trabajador para el logro de organizaciones efectivas y saludables. Por esta razón se espera que esta investigación demuestre el efecto que tienen las prácticas organizacionales positivas en los trabajadores y cómo estas se configuran 
entre sí. $\mathrm{Y}$ así las organizaciones en el contexto nacional consideren estos elementos como desafíos futuros y busquen de manera activa la realización de cambios pertinentes desde los fundamentos básicos, los cuales permitirían maximizar tanto los objetivos organizacionales como los de sus trabajadores.

\section{Consideraciones éticas}

El cuestionario integró un consentimiento informado de participación al inicio del instrumento. Como es producto de un proyecto de investigación se realizó una evaluación de los alcances éticos durante la evaluación previa a la adjudicación del proyecto.

\section{Contribución de los autores}

Ambos autores colaboraron en el desarrollo del escrito, el Dr. Jiménez encargándose principalmente de los antecedentes teóricos, y el Dr. Ossa de los análisis estadísticos y resultados.

\section{Financiación}

Este artículo se desarrolló en el marco del proyecto de Investigación Modelo sistémico de promoción en salud y calidad de vida para organizaciones educativas de las regiones de Nuble y Maule, con código 2050251 IF/ R, Dirección de Investigación, Universidad del Bío-Bío, Chile.

\section{Conflictos de interés}

No existen conflictos de interés asociado al desarrollo de la investigación y de la presentación de artículo.

\section{Referencias}

Acosta, H., Cruz, V., Salanova, M, \& Llorens, S. (2015). Healthy organization: Analyzing its meaning based on the HERO Model / Organizaciones saludables: analizando su significado desde el Modelo HERO. Revista de Psicologia Social / International Journal of Social Psychology, 30(2), 323-350. http://dx.doi.org/10.1080/2171 1976.2015.1016751

Alonso, F. (2014). Una panorámica de la salud mental de los profesores. Revista Iberoamericana de Educación, 66, 19-30. https://doi.org/10.35362/rie660375

Arenas, F., \& Andrade, V. (2013). Factores de riesgo psicosocial y compromiso (engagement) con el trabajo en una organización del sector salud de la ciudad de Cali, Colombia. Acta Colombiana de Psicología, 16(1), 43-56. http s://actacolombianapsicologia.ucatolica.edu.co/article/view/247

Ávila, M., Portalanza, C., \& Duque, E. (2017). Evaluación del engagement en trabajadores de una institución de educación superior en Ecuador. Revista cientifica Ecociencia, 4(4), 1-25. https://doi.org/10.21855/ecociencia. 44.43

Bakker, A., \& Rodriguez-Mun\#oz, A. (2012). Introduccio\#n a la psicologi\#a de la salud ocupacional positiva [Positive Occupational Health Psychology: An introduction]. Psicothema, 24, 62-65. http://www.psicothema.com/pdf /3979.pdf

Bakker, A., Albrecht, S., \& Leiter, M. (2011). Key questions regarding work engagement. European Journal of Work and Organizational Psychology 20, 4-48. https://doi.org/10.1080/1359432X.2010.485352 
Bedoya, M., Carrillo, L., Severiche, S. \& Espinosa, F. (2018). Factores asociados a la satisfacción laboral en docentes de una institución de educación superior del Caribe Colombiano. Revista Espacios, 39(2), 798-1015. https://www .revistaespacios.com/a18v39n02/a18v39n02p01.pdf

Bodero, H. (2014). El impacto de la calidad educativa. Apuntes de Ciencia \& Sociedad, 4(1), 112-117. https://doi.or $\mathrm{g} / 10.18259 / \mathrm{acs} .2014011$

Canales-Vergara, M., Valenzuela-Suazo, S., \& Luengo-Machuca., L. (2018). Calidad de vida en el trabajo en profesores de colegios públicos de Concepción, Chile. Enfermería Universitaria, 15(4), 370-382. https://doi.org/10.2220 1/eneo.23958421e.2018.4.544

Cánovas, F., \& Chiclana, C. (2018). Engagement, una plataforma para el desarrollo de la persona. Comunicación y hombre, 14, 55-64. https://www.redalyc.org/pdf/1294/129453532003.pdf

Carmona, M., Schaufeli, W., \& Salanova, M. (2019). The utrecht work engagement scale for students (UWES9S): Factorial validity, reliability, and measurement invariance in a Chilean sample of undergraduate university students. Frontiers in psychology, 10, 1017. https://doi.org/10.3389/fpsyg.2019.01017

Castilla-Gutiérrez, S., Colihuil-Catrileo, R., Bruneau-Chávez, J., \& Lagos-Hernández, R. (2021). Carga laboral y efectos en la calidad de vida de docentes universitarios y de enseñanza media. Chakiñan, Revista de Ciencias Sociales y Humanidades. https://chakinan.unach.edu.ec/index.php/chakinan/article/view/533

Chávez, D., Moreno, M., Beltrán, C. \& Perales, C. (2014). Burnout y Work Engagement en Docentes Universitarios de Zacatecas. Ciencia \& trabajo, 16(50), 116-120. http://dx.doi.org/10.4067/S0718-24492014000200010

Contreras, C. (2015). Determinación del nivel de Engagement laboral en trabajadores de una planta de producción de petróleo y gas costa afuera en México. Ciencia \& trabajo, 17(52), 37-42. http://dx.doi.org/10.4067/S071824492015000100008

Cruz, M. (2019). Análisis de estrategias para la fidelización del personal en empresas de transferencia de fondos: una revisión de la literatura científica. Trabajo de investigación. Repositorio de la Universidad Privada del Norte. ht tp://hdl.handle.net/11537/23138

Cuadra, D., Jorquera, R. \& Pérez, M. (2015). Las teorías subjetivas del profesor acerca de su salud laboral: Implicancias en la promoción de la salud preventiva en el trabajo docente. Ciencia y Trabajo, 17(52), 1-6. http://dx.doi.org/ $10.4067 /$ S0718-24492015000100002

Eduglobal (2012). Primer censo docente Chile 2012. Documento online. http://educacion2020.cl/documentos/prim er-censo-docente-chile-2012/

Erazo A. (2015). Un enfoque sistémico para comprender y mejorar los sistemas de salud. Revista Panamericana de Salud Pública, 38(3), 248-253. https://www.scielosp.org/article/rpsp/2015.v38n3/248-253/

Forbes, R. (2013). La psicología organizacional positiva y la mejora organizacional. Revista Éxito Empresarial, 227(1), 1-3. http://www.cegesti.org/exitoempresarial/publicaciones/publicacion_227_040313_es.pdf

Fundación Chile (2018). Engagement. Medición 2018. https://fch.cl/wp-content/uploads/2019/07/engagement-20 18.pdf

Garbanzo, G. (2015). Desarrollo organizacional y los procesos de cambio en las instituciones educativas, un reto de la gestión de la educación. Revista Educación, 40(1), 67-87. http://dx.doi.org/10.15517/revedu.v40i1.22534

García, F., Juárez, S., \& Salgado, L. (2018). Gestión escolar y calidad educativa. Revista Cubana de Educación Superior, 37(2), 206-216. http://scielo.sld.cu/scielo.php?script=sci_arttext\&pid=S0257-43142018000200016\&lng=es \&tlng=es.

García, M., González, R., Aldrete, M., Acosta, M., \& León, S. (2014). Relación entre calidad de vida en el trabajo y síntomas de estrés en el personal administrativo universitario. Ciencia y Trabajo, 16(50), 97-102. http://dx.doi .org/10.4067/S0718-24492014000200007

Gómez-Restrepo, C., Padilla, A., Rodríguez, V., Guzmán, J., Mejía, G., Avella-García, C. B., \& Edery, E. G. (2010). Influencia de la violencia en el medio escolar y en sus docentes: estudio en una localidad de Bogotá, Colombia. Revista Colombiana de Psiquiatría, 39(1), 22-44. . ISSN 0034-7450. 
González, R. C., Aranda, D. R., \& Berrocal, P. F. (2010). Docentes emocionalmente inteligentes. Revista electrónica interuniversitaria de formación del profesorado, 13(1), 41-49. http://dx.doi.org/10.4067/S0718-070520210001 00219

González, Y. (2020). Carga mental de trabajo percibida por profesores de establecimientos educacionales de la Región del Maule, Chile. Tesis para optar al grado de Magíster en Enfemería. Universidad Católica del Maule. Chile.

Guerrero-Barona, E., Gómez, R., Moreno-Manso, J. M., \& Guerrero-Molina, M. (2018). Factores de riesgo psicosocial, estrés percibido y salud mental en el profesorado. Psychosocial risk factors, perceived stress and mental health in teachers. Revista Clínica Contemporánea, 9(e2), 1-12. https://doi.org/10.5093/cc2018a2

Gutiérrez, M., Tomás, J., \& Pastor, A. (2020). Apoyo social de familia, profesorado y amigos, ajuste escolar y bienestar subjetivo en adolescentes peruanos. Suma Psicológica, 28(1), 17-24. https://doi.org/10.14349/sumapsi.2021.v 28.n1.3

Heckmann, B., Sandoval, F., \& Arroyo-Jofré, P. (2021). El ejercicio físico como factor protector de estrés en profesores de educación primaria y secundaria: Revisión Sistemática. Revista Horizonte Ciencias de la Actividad Física, 12(1), 70-89. http://revistahorizonte.ulagos.cl/index.php/horizonte/article/view/196

Hipólito, M., Masson, V., Monteiro, M., \& Gutiérrez G. (2017). Quality of working life: assessment of intervention studies. Rev Bras Enferm [Internet], 70(1), 178-186. http://dx.doi.org/10.1590/0034-7167-2015-0069

Horn, A., \& Murillo F. (2016). Incidencia de la dirección escolar sobre el compromiso de los docentes: Un estudio multinivel. Psicoperspectivas individuo y sociedad, 15(2), 64-77. https://www.psicoperspectivas.cl/index.php/ps icoperspectivas/article/viewFile/746/500

Jiménez, A., Jara, M. \& Miranda, E. (2012). Burnout, apoyo social y satisfacción laboral en docentes. Psicologia Escolar e Educacional, 16(1), 125-134. https://dx.doi.org/10.1590/S1413-85572012000100013

Jomuad, P., Antiquina, L., Cericos, E., Bacus, J., Vallejo, J., Dionio, B., et al. (2021). Teachers' workload in relation to burnout and work performance. International Journal of Educational Policy Research and Review. 8(2), 48-53. $\mathrm{h}$ ttps://journalissues.org/ijeprr/abstract/teachers-workload-in-relation-to-burnout-and-work-performance/

Knight, C., Patterson, M., \& Dawson, J. (2016). Building work engagement: A systematic review and meta-analysis investigating the effectiveness of work engagement interventions. Journal of Organizational Behavior, 38(6), 121. https://doi.org/10.1002/job.2167

Larrosa, F. (2010). Vocación docente versus profesión docente en las organizaciones educativas. Revista Electrónica Interuniversitaria de Formación del Profesorado, 13(4), 43-51. http://www.redalyc.org/articulo.oa?id=217015 570004

Ling, O., Fang, J., Brought, P., Qin, C., Bakker, A., Kalliath, T., O’Driscoll, M. et al. (2010). Role resources and workfamily enrichment: The role of work engagement. Journal of Vocational Behavior, 77, 470-480. https://doi.org /10.1016/j.jvb.2010.06.007

Lorente, L. \& Vera, M. (2010). Las organizaciones saludables: El engagement en el trabajo. Gestión práctica de riesgos laborales: Integración y desarrollo de la gestión de la prevención, 73, 16-20. https://pdfs.wke.es/1/5/4/2/pd00 00051542.pdf

Marsollier, R. (2021). Burnout y engagement en el análisis organizacional. Contribuciones y desafíos. Psicología UNEMI, 5(8), 62-71. https://doi.org/10.29076/issn.2602-8379vol5iss8.2021pp62-71p

Martínez, I., Youssef-Morgan, C., Chambel, M., \& Marques-Pinto, A. (2019). Antecedents of academic performance of university students: academic engagement and psychological capital resources. Educational Psychology, 39(8), 1047-1067. https://doi.org/10.1080/01443410.2019.1623382

Miño, A. (2016). Calidad de vida laboral en docentes chilenos. Summa Psicológica, 13(2), 45-55. https://doi.org/10 .18774 /summa-vol13.num2-256

Ospina, J. L., \& Meneghel, I. (2016). Clima organizacional como antecedente del Engagement en una muestra de empresas colombianas. Revista Interamericana de Psicologia Ocupacional, 35(1), 21-34. https://doi.org/10.217 72/ripo.v35n1a02 
Ossa, C., Quintana, I. \& Mendoza, R. (2015). Construyendo un modelo de Calidad de Vida Escolar. En: E. Saavedra et al. (Eds.) Resiliencia y calidad de vida. La Psicología educacional en diálogo con otras disciplinas. Talca: Ed. Universidad Católica del Maule.

Parada, M. \& Pérez, C. (2014). Relación del engagement académico con características académicas y socioafectivas en estudiantes de Odontología. Educación Médica Superior, 28(2), 199-215. Recuperado el 26 de febrero de 2021 de http://scielo.sld.cu/scielo.php?script=sci_arttext\&pid=S0864-21412014000200003\&lng=es\&tlng=es

Patlán, J. (2016). Construcción y propiedades psicométricas de la escala de calidad de vida en el trabajo. Ciencia y Trabajo, 18(56), 94-105. http://dx.doi.org/10.4067/S0718-24492016000200004

Pérez-Zapata, D., Peralta-Montecinos, J., \& Fernández-Dávila, P. (2014). Influencia de variables organizacionales en la calidad de vida laboral de funcionarios del sector público de salud en el extremo norte de Chile. Universitas Psychologica, 13(2), 541-551. https://doi.org/10.11144/Javeriana.UPSY13-2.ivoc

Pujol-Cols, L. \& Arraigada, M. (2018). Propiedades psicométricas de la versión corta de la Utrecht Work Engagement Scale en trabajadores argentinos. Pensamiento Psicológico, 16(2), 31-45. https://doi.org/10.11144/Javerianacal i.PPSI16-2.ppvc

Redondo, A. I. (2015). Engagement y clima organizacional en cooperativas. En VII Congreso Internacional de Investigación y Práctica Profesional en Psicología XXII Jornadas de Investigación XI Encuentro de Investigadores en Psicología del MERCOSUR. Facultad de Psicología, Universidad de Buenos Aires.

Ribeiro, B., Dos Santos, S., Scorsolini-Comin, F., \& Dalri, R. (2020). Being a professor in the context of the COVID-19 pandemic: reflections on mental health. Index de Enfermería, 29(3), 137-141. Recuperado el 06 de julio de 2021 de http://scielo.isciii.es/scielo.php?script=sci_arttext\&pid=S1132-12962020000200008\&lng=es\&tlng=en

Rubio, L. A. (2018). Justicia organizacional y engagement en docentes de una universidad privada de Armenia, Quindío. Contexto, 7, 47-56. https://doi.org/10.18634/ctxj.7v.0i.884

Ruiz de Chávez, D., Pando, M., Aranda, C., \& Almeida, C. (2014). Burnout y work engagement en docentes universitarios de Zacatecas. Ciencia \& trabajo, 16(50), 116-120. http://dx.doi.org/10.4067/S0718-24492014 000200010

Salanova, M. \& Llorens, S. (2016). Hacia una psicología positiva aplicada. Papeles del Psicólogo, 37(3), 161-164. htt p://www.redalyc.org/articulo.oa?id=77847916001

Salanova, M., Llorens, S., Cifre, E., \& Martínez, E. (2012). We Need a Hero! Toward a validation of the Healthy and Resilient Organization (HERO) Model. Group \& Organization Management,37(6), 785-822. https://doi.org/ $0.1177 / 1059601112470405$

Salanova, M., Martínez, I., \& Llorens, S. (2014). Una mirada más positiva a la salud ocupacional desde la Psicología organizacional positiva en tiempos de crisis: equipo de investigación WONT. Papeles del psicólogo, 35(1), 22-30. http://www.papelesdelpsicologo.es/pdf/2318.pdf

Schaufeli, W., \& De Witte, H. (2017). Work Engagement: Real or Redundant? Burnout Research, 5, 1-2. https://do i.org/10.1016/j.burn.2017.06.001

Silva, L., Picinin, C., Pilati, L. \& Franco, A. (2021). Work-life balance in Higher Education: A systematic review of the impact on the well-being of teachers. Ensaio: Avaliação e Politicas Públicas em Educação, 29(112), 691-717. htt ps://doi.org/10.1590/S0104-403620210002903021

Superintendencia de Seguridad Social (2017). Informe anual Estadisticas de Seguridad Social. http://www.suseso.gob .cl/607/articles-496701_archivo_01.pdf

Tabachnick, B. \& Fidell, L. (2001). Using multivariate statistics, (4 ed.). Nueva York: Harper \& Row.

Toscano-del Cairo, C., Vesga-Rodríguez, J., \& Avendaño-Prieto, B. (2020). Quality of life at work and its relationship with engagement. Acta Colombiana de Psicologia, 23(1), 128-137. http://www.doi.org/10.14718/ACP.2020.2 3.1.7

Van den Berg, J., Mastenbroek, N., Scheepers R., \& Jaarsma D. (2017). Work engagement in health professions education. Medical Teacher, 39(11), 1110-1118. https://doi.org/10.1080/0142159X.2017.1359522 
Ventura, M. Salanova, M., \& Llorens, S. (2015). Professional self-efficacy as a predictor of burnout and engagement: The role of challenge and hindrance demands. The Journal of Psychology, 149(3), 277-302. https://doi.org/10. 1080/00223980.2013.876380

Vesga, J., García, M., Forero, C., Toscano, C., Quiroz, E., \& Pazmay, S. (2021). La relación entre la edad, el engagement y la disposición al cambio organizacional. Interdisciplinaria, 38(2), 224-241. https://doi.org/10.16888/interd. 2021.38.2.15

\section{Notas}

* Artículo de investigación

\section{Licencia Creative Commons CC BY 4.0}

Para citar este artículo: Ossa, C., \& Jiménez, A. (2021). Relación entre engagement y calidad de vida en el trabajo en funcionarios de establecimientos educacionales chilenos. Cuadernos de Administración, 34. https ://doi.org/10.11144/Javeriana.cao34.recvtf 